\title{
On the Exponential Radon Transform and Its Extension to Certain Functions Spaces
}

\author{
S. K. Q. Al-Omari ${ }^{1}$ and A. Kılıçman ${ }^{2}$ \\ ${ }^{1}$ Department of Applied Sciences, Faculty of Engineering Technology, Al-Balqa Applied University, Amman 11134, Jordan \\ ${ }^{2}$ Department of Mathematics and Institute for Mathematical Research, University Putra Malaysia (UPM), \\ 43400 Serdang, Selangor, Malaysia
}

Correspondence should be addressed to A. Kılıçman; akilic@upm.edu.my

Received 25 October 2013; Accepted 18 April 2014; Published 5 May 2014

Academic Editor: Abdullah Alotaibi

Copyright (c) 2014 S. K. Q. Al-Omari and A. Kılıçman. This is an open access article distributed under the Creative Commons Attribution License, which permits unrestricted use, distribution, and reproduction in any medium, provided the original work is properly cited.

We investigate the exponential Radon transform on a certain function space of generalized functions. We establish certain space of generalized functions for the cited transform. The transform that is obtained is well defined. More properties of consistency, convolution, analyticity, continuity, and sufficient theorems have been established.

\section{Introduction}

The Radon transform of a sufficiently nice function $f$ defined on $\mathbb{R}^{n}$ is given by

$$
\left(\mathscr{R}_{\vartheta} f\right)(\eta) \equiv(\mathscr{R} f)(\vartheta, \eta) \equiv \int_{\vartheta^{\perp}} f(\eta \vartheta+u) \mathbf{d} u,
$$

where $(\vartheta, \eta) \in \widetilde{\mathbb{R}}^{n}=\sum_{n-1} x p, \sum_{n-1}$ is the unit sphere in $\mathbb{R}^{n}$, and $\mathbf{d} u$ is the Euclidean measure on the subspace $\vartheta^{\perp}$ orthogonal to $\vartheta$.

Applications of the Radon transform occur in a number of areas, such as seismic signal processing, remote sensing, and system identification from output data $[1,2]$. The Radon transform is extended to various spaces of distributions, rapidly decreasing and integrable Boehmians $[3,4]$. More about the Radon transform is given in [5-9].

The discrete Radon transform is defined by $[10,11]$. The attenuated Radon transform is defined in Mikusiński et al. $[12,13]$. For a uniform attenuation coefficient $\mu \in \mathbb{C}$, the exponential Radon transform of a compactly supported real valued function $f$, defined on $\mathbb{R}^{2}$, is given by Kurusa and Hertle $[7,8]$ :

$$
\mathbf{T}_{\mu}^{e} f(\vartheta, t)=\int_{\mathbb{R}^{2}} f(\mathbf{x}) \delta(\mathbf{x} \cdot \vartheta-t) e^{\mu \mathbf{x} \cdot \vartheta^{\perp}} \mathbf{d} \mathbf{x}, \quad t \in \mathbb{R},
$$

where $\mathcal{Y}=(\cos \varphi, \sin \varphi)^{\tau}$ is a unit vector on $\mathcal{S}^{1}, \varphi \in$ $[0,2 \pi), \vartheta^{\perp}=(-\sin \varphi, \cos \varphi)$.

The exponential Radon transform constitutes a mathematical model for imaging modalities such as X-ray tomography for $\mu=0$, single photon emission tomography for $\mu \in \mathbb{R}$, and optical polarization tomography of trass tensor field [14]. However, if in addition $\mu$ is unknown, then one first must find $\mu$ and then find $f$. This is the identification problem.

The exponential Radon transform, as a generalization of the Radon transform, is defined as a mapping of function spaces and is also represented in terms of Fourier transforms of its domain and range, and this leads to a characterization of the range of the transform. For more information about the exponential Radon transform, we refer to $[15,16]$.

\section{General Construction of Boehmians}

The minimal structure necessary for the construction of Boehmians consists of the following elements:

(i) a set a and a commutative semigroup (g, *);

(ii) an operation $\odot: \mathbf{a} \times \mathbf{g} \rightarrow \mathbf{a}$ such that for each $x \in \mathbf{a}$ and $v_{1}, v_{2}, \in \mathbf{g}$,

$$
x \odot\left(v_{1} * v_{2}\right)=\left(x \odot v_{1}\right) \odot v_{2} ;
$$


(iii) a collection $\Delta \subset \mathbf{g}^{\mathbb{N}}$ satisfying the following:

(1) if $x, y \in \mathbf{a},\left(v_{n}\right) \in \Delta, x \odot v_{n}=y \odot v_{n}$ for all $n$, then $x=y$;

(2) if $\left(v_{n}\right),\left(\sigma_{n}\right) \in \Delta$, then $\left(v_{n} * \sigma_{n}\right) \in \Delta, \Delta$ being the set of all delta sequences.

Consider

$$
\begin{gathered}
\mathscr{A}=\left\{\left(x_{n}, v_{n}\right): x_{n} \in \mathbf{a},\left(v_{n}\right) \in \Delta, x_{n} \odot v_{m}\right. \\
\left.=x_{m} \odot v_{n}, \forall m, n \in \mathbb{N}\right\} .
\end{gathered}
$$

If $\left(x_{n}, v_{n}\right),\left(y_{n}, \sigma_{n}\right) \in \mathscr{A}, x_{n} \odot \sigma_{m}=y_{m} \odot v_{n}, \forall m, n \in \mathbb{N}$, then we say $\left(x_{n}, v_{n}\right) \sim\left(y_{n}, \sigma_{n}\right)$. The relation $\sim$ is an equivalence relation in $\mathscr{A}$. The space of equivalence classes in $\mathscr{A}$ is denoted by $\boldsymbol{\delta}(\mathbf{a},(\mathbf{g}, *), \odot, \Delta)$. Elements of $\boldsymbol{\delta}(\mathbf{a},(\mathbf{g}, *), \odot, \Delta)$ are called Boehmians.

Between a and $\boldsymbol{\delta}(\mathbf{a},(\mathbf{g}, *), \odot, \Delta)$ there is a canonical embedding expressed as

$$
x \longrightarrow \frac{x \odot s_{n}}{s_{n}} \text { as } n \longrightarrow \infty .
$$
by

The operation $\odot$ can be extended to $\boldsymbol{\delta}(\mathbf{a},(\mathbf{g}, *), \odot, \Delta) \times \mathbf{a}$

$$
\frac{x_{n}}{v_{n}} \odot t=\frac{x_{n} \odot t}{v_{n}} .
$$

In $\boldsymbol{\delta}(\mathbf{a},(\mathbf{g}, *), \Delta)$, two types of convergence exist:

(1) a sequence $\left(h_{n}\right)$ in $\boldsymbol{\delta}(\mathbf{a},(\mathbf{g}, *), \odot, \Delta)$ is said to be $\delta$ convergent to $h$ in $\boldsymbol{\delta}(\mathbf{a},(\mathbf{g}, *), \odot, \Delta)$, denoted by $h_{n} \stackrel{\delta}{\rightarrow}$ $h$ as $n \rightarrow \infty$, if there exists a delta sequence $\left(v_{n}\right)$ such that $\left(h_{n} \odot v_{n}\right),\left(h \odot v_{n}\right) \in \mathbf{a}, \forall k, n \in \mathbb{N}$, and $\left(h_{n} \odot v_{k}\right) \rightarrow\left(h \odot v_{k}\right)$ as $n \rightarrow \infty$, in a, for every $k \in \mathbb{N}$;

(2) a sequence $\left(h_{n}\right)$ in $\boldsymbol{\delta}(\mathbf{a},(\mathbf{g}, *), \odot, \Delta)$ is said to be $\Delta$ convergent to $h$ in $\delta(\mathbf{a},(\mathbf{g}, *), \odot, \Delta)$, denoted by $h_{n} \stackrel{\Delta}{\rightarrow}$ $h$ as $n \rightarrow \infty$, if there exists a $\left(v_{n}\right) \in \Delta$ such that $\left(h_{n}-\right.$ $h) \odot v_{n} \in \mathbf{a}, \forall n \in \mathbb{N}$, and $\left(h_{n}-h\right) \odot v_{n} \rightarrow 0$ as $n \rightarrow \infty$ in $\mathbf{a}$.

The following theorem is equivalent to the statement of $\delta$-convergence.

Theorem 1. $h_{n} \stackrel{\delta}{\rightarrow} h(n \rightarrow \infty)$ in $\boldsymbol{\delta}(\mathbf{a},(\mathbf{g}, *), \odot, \Delta)$ if and only if there is $f_{n, k}, f_{k} \in \mathbf{a}$ and $v_{k} \in \Delta$ such that $h_{n}=\left[f_{n, k} / v_{k}\right]$, $h=\left[f_{k} / v_{k}\right]$ and for each $k \in \mathbb{N}, f_{n, k} \rightarrow f_{k}$ as $n \rightarrow \infty$ in a.

For further discussion see [3, 17-21].

\section{Necessary and Sufficient Conditions}

Denote by $\mathbf{l}^{1}\left(\mathcal{S}^{1} \times \mathbb{R}\right)$ the space of Lebesgue complex-valued measurable functions of bounded support defined on $\mathcal{S}^{1} \times \mathbb{R}$ and satisfying

$$
\||\psi((\vartheta, t))|\|=\int_{\mathcal{S}^{1}} \int_{\mathbb{R}}|\psi((\vartheta, t))|^{2} e^{\mu \mathbf{y} \cdot \vartheta^{\perp}} \mathbf{d} \vartheta \mathbf{d} t<\infty
$$

then $\mathbf{T}_{\mu}^{e} \psi \in \mathbf{l}^{1}\left(\mathcal{S}^{1} \times \mathbb{R}\right), \mu \in \mathbb{R}, \mathbf{y} \in \mathbb{R}^{2}$ being arbitrary but fixed.

By $\boldsymbol{\kappa}\left(\mathbb{R}^{2}\right)$ denote the space of test functions of bounded support defined on $\mathbb{R}^{2}$.

Let $\Delta$ be the set of sequences $\left(\mu_{n}(\mathbf{x})\right) \in \boldsymbol{\kappa}\left(\mathbb{R}^{2}\right)$ such that $[3,(2.6)-(2.8)]$

$$
\begin{gathered}
\int_{\mathbb{R}^{2}} \mu n(\mathbf{x}) \mathbf{d} \mathbf{x}=1, \\
\int_{\mathbb{R}^{2}}|\mu n(\mathbf{x})| \mathbf{d} \mathbf{x}<M, \quad 0<M \in \mathbb{R}, \\
\operatorname{supp}\left(\mu_{n}(\mathbf{x})\right) \longrightarrow 0 \quad \text { as } n \longrightarrow \infty .
\end{gathered}
$$

The convolution product between two functions is defined by the integral equation

$$
(f * g)(\mathbf{x})=\int_{\mathbb{R}^{2}} f(\mathbf{x}-\mathbf{y}) g(\mathbf{y}) \mathbf{d y},
$$

where $\mathbf{x} \in \mathbb{R}^{2}$.

Now we construct the space $\boldsymbol{\delta}\left(\mathbf{1}^{1},(\boldsymbol{\kappa}, *), \times, \Delta\right)$ of Boehmians.

We have the following definition.

Definition 2. Let $\psi \in \mathbf{l}^{1}\left(\mathcal{S}^{1} \times \mathbb{R}\right)$ and $\xi \in \boldsymbol{\kappa}\left(\mathbb{R}^{2}\right)$; then we define the mapping $\times$ as

$$
(\psi \times \xi)(\vartheta, t)=\int_{\mathbb{R}^{2}} \psi(\vartheta, t-\mathbf{y} \cdot \vartheta) \xi(\mathbf{y}) e^{\mu \mathbf{y} \cdot \vartheta^{\perp}} \mathbf{d y}
$$

$$
(\vartheta, t) \in \mathcal{S}^{1} \times \mathbb{R} \text {. }
$$

Theorem 3. Let $\psi \in \mathbf{l}^{1}\left(\mathcal{S}^{1} \times \mathbb{R}\right)$ and $\xi \in \boldsymbol{\kappa}\left(\mathbb{R}^{2}\right)$; then $\psi \times \xi \in$ $\mathbf{l}^{1}\left(\mathcal{S}^{1} \times \mathbb{R}\right)$.

Proof. Let $\psi \in \mathbf{l}^{1}\left(\mathcal{S}^{1} \times \mathbb{R}\right)$ and $\xi \in \boldsymbol{\kappa}\left(\mathbb{R}^{2}\right)$. By using (10), Fubini's theorem, and Jensen's inequality we get

$$
\begin{aligned}
& \|\|(\psi \times \xi)(\vartheta, t) \mid \|^{2} \\
& =\int_{\mathcal{S}^{1}} \int_{\mathbb{R}}|(\psi \times \xi)(\vartheta, t)|^{2} \mathbf{d} \vartheta \mathbf{d} t \\
& =\int_{\mathcal{S}^{1}} \int_{\mathbb{R}}\left|\int_{\mathbb{R}^{2}} \psi(\vartheta, t-\mathbf{y} \cdot \theta) \xi(\mathbf{y}) e^{\mu \mathbf{y} \cdot \vartheta^{\perp}} \mathbf{d y}\right|^{2} \mathbf{d} \vartheta \mathbf{d} t \\
& =\int_{\mathbb{R}^{2}}|\xi(\mathbf{y})| \int_{\mathcal{S}^{1}} \int_{\mathbb{R}}|\psi(\vartheta, t-\mathbf{y} \cdot \vartheta)|^{2} e^{\mu \mathbf{y} \cdot \vartheta^{\perp}} \mathbf{d} \vartheta \mathbf{d} t \mathbf{d} \mathbf{y} \\
& <M\||\psi|\|^{2},
\end{aligned}
$$

where $M$ is a positive constant.

The proof is therefore completed.

Theorem 4. Let $\psi_{n} \rightarrow \psi$ in $\mathbf{l}^{1}\left(\mathcal{S}^{1} \times \mathbb{R}\right)$ and $\xi \in \boldsymbol{\kappa}\left(\mathbb{R}^{2}\right)$; then $\psi_{n} \times \xi \rightarrow \psi \times \xi$ as $n \rightarrow \infty$.

Proof of this theorem follows from Theorem 3.

Theorem 5. Let $\psi \in \mathbf{l}^{1}\left(\mathcal{S}^{1} \times \mathbb{R}\right)$ and $\xi_{1}, \xi_{2} \in \boldsymbol{\kappa}\left(\mathbb{R}^{2}\right)$; then one has

$$
\psi \times\left(\xi_{1} * \xi_{2}\right)=\left(\psi \times \xi_{1}\right) \times \xi_{2}
$$


Proof. Let $(\vartheta, t) \in \mathcal{S}^{1} \times \mathbb{R}$. Using (10) and (9) we write

$$
\begin{aligned}
(\psi & \left.\times\left(\xi_{1} * \xi_{2}\right)\right)(\vartheta, t) \\
& =\int_{\mathbb{R}^{2}} \psi(\vartheta, t-\mathbf{y} \cdot \vartheta)\left(\xi_{1} * \xi_{2}\right)(\mathbf{y}) e^{\mu \mathbf{y} \cdot \vartheta^{\perp}} \mathbf{d y} \\
& =\int_{\mathbb{R}^{2}} \psi(\vartheta, t-\mathbf{y} \cdot \vartheta)\left(\int_{\mathbb{R}^{2}} \xi_{1}(\mathbf{y}-\mathbf{x}) \xi_{2}(\mathbf{x}) \mathbf{d x}\right) e^{\mu \mathbf{y} \cdot \vartheta^{\perp}} \mathbf{d y} \\
& =\int_{\mathbb{R}^{2}}\left(\int_{\mathbb{R}^{2}} \psi(\vartheta, t-\mathbf{y} \cdot \vartheta) \xi_{1}(\mathbf{y}-\mathbf{x}) e^{\mu \mathbf{y} \cdot \vartheta^{\perp}} \mathbf{d y}\right) \xi_{2}(\mathbf{x}) \mathbf{d x} .
\end{aligned}
$$

The substitution $\mathbf{y}-\mathbf{x}=\mathbf{z}, \mathbf{y}, \mathbf{x}, \mathbf{z} \in \mathbb{R}^{2}$, implies

$$
\begin{aligned}
\left(\psi \times\left(\xi_{1} * \xi_{2}\right)\right)(\vartheta, t) & \\
=\int_{\mathbb{R}^{2}} & \left(\int_{\mathbb{R}^{2}} \psi(\vartheta, t-(\mathbf{z}+\mathbf{x}) \cdot \vartheta) \xi_{1}(\mathbf{z}) e^{\mu(\mathbf{z}+\mathbf{x}) \vartheta^{\perp}} \mathbf{d z}\right) \\
& \times \xi_{2}(\mathbf{x}) \mathbf{d} \mathbf{x} \\
= & \int_{\mathbb{R}^{2}}\left(\int_{\mathbb{R}^{2}} \psi(\vartheta,(t-\mathbf{x} \cdot \vartheta)-\mathbf{z} \cdot \vartheta) \xi_{1}(\mathbf{z}) e^{\mu \mathbf{x} \cdot \vartheta^{\perp}} \mathbf{d z}\right) \\
& \times \xi_{2}(\mathbf{x}) e^{\mu \mathbf{x} \cdot \vartheta^{\perp}} \mathbf{d x} \\
= & \int_{\mathbb{R}^{2}}\left(\psi \times \xi_{1}\right)(\vartheta, t-\mathbf{x} \cdot \vartheta) \xi_{2}(\mathbf{x}) e^{\mu \mathbf{x} \cdot \vartheta^{\perp}} \mathbf{d x} \\
= & \left(\left(\psi \times \xi_{1}\right) \times \xi_{2}\right)(\vartheta, t) .
\end{aligned}
$$

This completes the proof of the theorem.

Theorem 6. Let $\psi_{1}, \psi_{2} \in \mathbf{l}^{1}\left(\mathcal{S}^{1} \times \mathbb{R}\right)$ and $\xi \in \boldsymbol{\kappa}\left(\mathbb{R}^{2}\right)$; then

$$
\left(\psi_{1}+\psi_{2}\right) \times \xi=\psi_{1} \times \xi+\psi_{2} \times \xi_{2} .
$$

Theorem 7. Let $\alpha \in \mathbb{C}, \psi \in \mathbf{l}^{1}\left(\mathcal{S}^{1} \times \mathbb{R}\right)$ and $\xi \in \boldsymbol{\kappa}\left(\mathbb{R}^{2}\right)$; then

$$
\alpha(\psi \times \xi)=(\alpha \psi) \times \xi
$$

Proof of Theorems 6 and 7 follows from simple integration. Detailed proof is thus avoided.

Theorem 8. Let $\psi \in \mathbf{l}^{1}\left(\mathcal{S}^{1} \times \mathbb{R}\right)$ and $\left(\mu_{n}\right) \in \Delta$; then $\psi \times \mu_{n} \rightarrow$ $\psi$ as $n \rightarrow \infty$.

Proof. Let $\psi \in \mathbf{l}^{1}\left(\mathcal{S}^{1} \times \mathbb{R}\right)$. Since $\boldsymbol{\kappa}(\mathbb{R} \times \mathbb{R})$ is dense in $\mathbf{l}^{1}\left(\mathcal{S}^{1} \times \mathbb{R}\right)$, we can choose $\alpha \in \boldsymbol{\kappa}(\mathbb{R} \times \mathbb{R})$ such that

$$
\||\psi-\alpha|\|<\epsilon, \quad \epsilon>0
$$

From the analysis applied for proving Theorem 3 and by applying (17) we get

$$
\left\|\left|(\psi-\alpha) \times \mu_{n}\right|\right\| \leq M\||\psi-\alpha|\|<M \epsilon .
$$

Also, for each fixed $(\vartheta, t) \in \mathcal{S}^{1} \times \mathbb{R}$ define

$$
g(\mathbf{y})=\alpha(\vartheta, t-\mathbf{y} \cdot \vartheta) e^{\mu \mathbf{y} \cdot \vartheta^{\perp}}, \quad \mathbf{y} \in \mathbb{R}^{2}
$$

then $g(\mathbf{y}) \in \boldsymbol{\kappa}\left(\mathbb{R}^{2}\right)$ and hence $g(\mathbf{y})$ uniformly continuous on $\mathbb{R}^{2}$. Thus, there is $\delta>0$ such that $|g(\mathbf{y})-g(\mathbf{x})|<\epsilon$ whenever $|\mathbf{y}-\mathbf{x}| \leq \delta$.

Moreover supp $\alpha(\vartheta, t) \subseteq[a, b] \times \mathbb{k}, \mathbb{k} \subseteq \mathbb{R}-\{0\}$ implies $\alpha(\vartheta, t)=0, \forall(\vartheta, t) \notin[a-\delta, b+\delta] \times \mathbb{k}$.

Hence, by (8) and the fact that $|(g(y)-g(0))|<\epsilon$, by Jensen's inequality, we have

$$
\begin{aligned}
& \left\|\left|\left(\alpha \times \mu_{n}-\alpha\right)(\vartheta, t)\right|\right\|^{2} \\
& =\int_{\mathcal{S}^{1}} \int_{\mathbb{R}}\left|\left(\alpha \times \mu_{n}-\alpha\right)(\vartheta, t)\right|^{2} \mathbf{d} \vartheta \mathbf{d} t \\
& =\int_{\mathcal{S}^{1}} \int_{\mathbb{R}} \mid \int_{\mathbb{R}^{2}}\left(\alpha(\vartheta, t-\mathbf{y} \cdot \vartheta) \mu_{n}(\mathbf{y}) e^{\mu \mathbf{y} \cdot \vartheta^{\perp}}\right. \\
& \quad=\int_{\mathcal{S}^{1}} \int_{\mathbb{R}}\left|\int_{\mathbb{R}^{2}}(g(\mathbf{y})-g(0)) \mu_{n}(\mathbf{y}) \mathbf{d y}\right|^{2} \mathbf{d} \vartheta \mathbf{d} t \\
& \leq \int_{\mathbb{k}} \int_{a-\delta}^{b+\delta} \int_{\mathbb{R}^{2}}|(g(\mathbf{y})-g(0))|^{2}\left|\mu_{n}(\mathbf{y})\right| \mathbf{d y} \mathbf{d} \vartheta \mathbf{d} t \\
& \leq \epsilon^{2} \int_{\mathbb{k}} \int_{a-\delta}^{b+\delta} \int_{\mathbb{R}^{2}}\left|\mu_{n}(\mathbf{y})\right| \mathbf{d y} \mathbf{d} \vartheta \mathbf{d} t \\
& \quad=\epsilon^{2} M m\left(\left.\mathbb{d}\right|^{2} \mathbf{d} \vartheta \mathbf{d} t\right. \\
&
\end{aligned}
$$

where $m(\mathbb{k})$ is the Lebesgue measure of $\mathbb{k}$.

Hence, using (17), (18), and (20) we, for large values of $n$, get

$$
\begin{aligned}
\left\|\left|\psi \times \mu_{n}-\psi\right|\right\| \leq & \left\|\left|(\psi-\alpha) \times \mu_{n}\right|\right\| \\
& +\left\|\left|\alpha \times \mu_{n}-\alpha\right|\right\|+\||\alpha-\psi|\| \\
< & M \epsilon+\epsilon^{2} M m(\mathbb{k})(b-a+2 \delta)+\epsilon \\
= & \left(M+\epsilon^{2} M m(\mathbb{k})(b-a+2 \delta)+1\right) \epsilon .
\end{aligned}
$$

Hence $\psi \times \mu_{n} \rightarrow \psi$ as $n \rightarrow \infty$.

The Boehmian space $\boldsymbol{\delta}\left(\mathbf{1}^{1},(\boldsymbol{\kappa}, *), \times, \Delta\right)$ is constructed.

The sum and multiplication by a scalar of two Boehmians are naturally defined in the respective ways:

$$
\begin{gathered}
{\left[\frac{\left(f_{n}\right)}{\left(\phi_{n}\right)}\right]+\left[\frac{\left(g_{n}\right)}{\left(\tau_{n}\right)}\right]=\left[\frac{\left(f_{n} \times \tau_{n}\right)+\left(g_{n} \times \phi_{n}\right)}{\left(\phi_{n} * \tau_{n}\right)}\right],} \\
\eta\left[\frac{\left(f_{n}\right)}{\left(\phi_{n}\right)}\right]=\left[\frac{\left(\eta f_{n}\right)}{\left(\phi_{n}\right)}\right],
\end{gathered}
$$

$\eta$ being complex number.

The operations $\times$ and the derivative are defined by

$$
\begin{gathered}
{\left[\frac{\left(f_{n}\right)}{\left(\phi_{n}\right)}\right] \times\left[\frac{\left(g_{n}\right)}{\left(\tau_{n}\right)}\right]=\left[\frac{\left(f_{n} \times g_{n}\right)}{\left(\epsilon_{n} * \tau_{n}\right)}\right],} \\
\mathscr{D}^{\alpha}\left[\frac{\left(f_{n}\right)}{\left(\phi_{n}\right)}\right]=\left[\frac{\left(\mathscr{D}^{\alpha} f_{n}\right)}{\left(\phi_{n}\right)}\right] .
\end{gathered}
$$


Between $\mathbf{l}^{1}\left(\mathcal{S}^{1} \times \mathbb{R}\right)$ and $\boldsymbol{\delta}\left(\mathbf{l}^{1},(\boldsymbol{\kappa}, *), \times, \Delta\right)$ the canonical embedding admits

$$
f \longrightarrow\left[\frac{\left(f \times \phi_{n}\right)}{\left(\phi_{n}\right)}\right] \text { as } n \longrightarrow \infty .
$$

The operation $\times$ can be extended to $\delta\left(\mathbf{l}^{1},(\boldsymbol{\kappa}, *), \times, \Delta\right) \times$ $\mathbf{l}^{1}\left(\mathcal{S}^{1} \times \mathbb{R}\right)$ by

$$
\left[\frac{\left(f_{n}\right)}{\left(\phi_{n}\right)}\right] \times f=\left[\frac{\left(f_{n} \times f\right)}{\left(\phi_{n}\right)}\right] .
$$

By $\boldsymbol{\delta}\left(\mathbf{l}^{1},(\boldsymbol{\kappa}, *), *, \Delta\right)$ denote the corresponding Boehmian space obtained from $\mathbf{l}^{1}\left(\mathcal{S}^{1} \times \mathbb{R}\right), \boldsymbol{\kappa}\left(\mathbb{R}^{2}\right), \Delta$ and the product *.

Theorem 9. Let $\psi \in \mathbf{l}^{1}\left(\mathcal{S}^{1} \times \mathbb{R}\right)$ and $\xi \in \boldsymbol{\kappa}\left(\mathbb{R}^{2}\right)$; then

$$
\mathbf{T}_{\mu}^{e}(\psi * \xi)(\vartheta, t)=\left(\mathbf{T}_{\mu}^{e} \psi \times \xi\right)(\vartheta, t),(\vartheta, t) \in \mathcal{S}^{1} \times \mathbb{R} .
$$

Proof. Let $(\vartheta, t) \in\left(\mathcal{S}^{1} \times \mathbb{R}\right)$. By employing (2) for (9) we get

$$
\begin{aligned}
\mathbf{T}_{\mu}^{e} & (\psi * \xi)(\vartheta, t) \\
& =\int_{\mathbb{R}^{2}}(\psi * \xi)(\mathbf{x}) \delta(\mathbf{x} \cdot \vartheta-t) e^{\mu \mathbf{x} \cdot \vartheta^{\perp}} \mathbf{d x} \\
& =\int_{\mathbb{R}^{2}}\left(\int_{\mathbb{R}^{2}} \psi(\mathbf{x}-\mathbf{y}) \xi(\mathbf{y}) \mathbf{d y}\right) \delta(\mathbf{x} \cdot \vartheta-t) e^{\mu \mathbf{x} \cdot \vartheta^{\perp}} \mathbf{d} \mathbf{x} \\
& =\int_{\mathbb{R}^{2}}\left(\int_{\mathbb{R}^{2}} \psi(\mathbf{x}-\mathbf{y}) \delta(\mathbf{x} \cdot \vartheta-t) e^{\mu \mathbf{x} \cdot \vartheta^{\perp}} \mathbf{d x}\right) \xi(\mathbf{y}) \mathbf{d y} .
\end{aligned}
$$

The substitution $\mathbf{x}-\mathbf{y}=\mathbf{z}$ implies $\mathbf{x}=\mathbf{y}+\mathbf{z}$ and $\mathbf{d x}=\mathbf{d z}$. Thus we get

$$
\begin{aligned}
\mathbf{T}_{\mu}^{e} & (\psi * \xi)(\vartheta, t) \\
& =\int_{\mathbb{R}^{2}} \int_{\mathbb{R}^{2}} \psi(\mathbf{z}) \delta(\mathbf{z} \cdot \vartheta-(t-\mathbf{y} \vartheta)) e^{\mu \mathbf{z} \cdot \vartheta^{\perp}} e^{\mu \mathbf{y} \cdot \vartheta^{\perp}} \mathbf{d z} \xi(\mathbf{y}) \mathbf{d y} \\
& =\int_{\mathbb{R}^{2}} \int_{\mathbb{R}^{2}} \mathbf{T}_{\mu}^{e} \psi(t-\mathbf{y} \cdot \vartheta) e^{\mu \mathbf{y} \cdot \vartheta^{\perp}} \xi(\mathbf{y}) \mathbf{d y} \\
& =\left(\mathbf{T}_{\mu}^{e} \psi \times \xi\right)(\vartheta, t) .
\end{aligned}
$$

This completes the proof of the theorem.

\section{The Exponential Radon Transform of Boehmians}

Definition 10. Let $\beta_{n}=\left[\psi_{n} / \mu_{n}\right] \in \boldsymbol{\delta}\left(\mathbf{l}^{1},(\boldsymbol{\kappa}, *), *, \Delta\right)$; then we define its exponential Radon transform as the mapping

$$
\overrightarrow{\mathbf{T}_{\mu}^{e}}\left[\frac{\psi_{n}}{\mu_{n}}\right]=\left[\frac{\mathbf{T}_{\mu}^{e} \psi_{n}}{\mu_{n}}\right]
$$

in the space $\boldsymbol{\delta}\left(\mathbf{1}^{1},(\boldsymbol{\kappa}, *), \times, \Delta\right)$.
Definition 10 is well defined by Theorem 9.

To show that (29) is well defined, let $\left[\psi_{n} / \mu_{n}\right],\left[\xi_{n} / \epsilon_{n}\right] \in$ $\boldsymbol{\delta}\left(\mathbf{1}^{1},(\boldsymbol{\kappa}, *), *, \Delta\right)$ and $\left[\psi_{n} / \mu_{n}\right]=\left[\xi_{n} / \epsilon_{n}\right]$; then

$$
\psi_{n} * \epsilon_{m}=\xi_{m} * \mu_{n}
$$

Employing $\mathbf{T}_{\mu}^{e}$ for (30) and using Theorem 9 imply that

$$
\mathbf{T}_{\mu}^{e} \psi_{n} \times \epsilon_{m}=\mathbf{T}_{\mu}^{e} \xi_{m} \times \mu_{n} .
$$

From (31) we see that $\mathbf{T}_{\mu}^{e} \psi_{n} / \mu_{n} \sim \mathbf{T}_{\mu}^{e} \xi_{n} / \epsilon_{n}$ in the sense of $\boldsymbol{\delta}\left(\mathbf{l}^{1},(\boldsymbol{\kappa}, *), \times, \Delta\right)$.

This completes the proof of the theorem.

Theorem 11. Let $\beta_{1}, \beta_{2} \in \boldsymbol{\delta}\left(\mathbf{l}^{1},(\boldsymbol{\kappa}, *), *, \Delta\right)$; then $\overrightarrow{\mathbf{T}_{\mu}^{e}}\left(\beta_{1} * \beta_{1}\right)=$ $\overrightarrow{\mathbf{T}_{\mu}^{e}} \beta_{1} \times \beta_{2}$.

Proof. Assume the requirements of the theorem are satisfied for some $\beta_{1}, \beta_{2} \in \boldsymbol{\delta}\left(\mathbf{l}^{1},(\boldsymbol{\kappa}, *), *, \Delta\right)$; then there are $\left(f_{n}\right),\left(\kappa_{n}\right) \in$ $\mathbf{l}^{1}\left(\mathcal{S}^{1} \times \mathbb{R}\right)$ and $\left(\varphi_{n}\right),\left(\phi_{n}\right) \in \Delta$ such that $\beta_{1}=\left[\left(f_{n}\right) /\left(\varphi_{n}\right)\right]$ and $\beta_{2}=\left[\left(\kappa_{n}\right) /\left(\phi_{n}\right)\right]$. Therefore, we write

$$
\begin{aligned}
\overrightarrow{\mathbf{T}_{\mu}^{e}} & \left(\beta_{1} * \beta_{2}\right) \\
= & \overrightarrow{\mathbf{T}_{\mu}^{e}}\left(\left[\frac{\left(f_{n}\right) *\left(\kappa_{n}\right)}{\left(\varphi_{n}\right) *\left(\phi_{n}\right)}\right]\right)=\left[\frac{\mathbf{T}_{\mu}^{e}\left(\left(f_{n}\right) *\left(\kappa_{n}\right)\right)}{\left(\varphi_{n}\right) *\left(\phi_{n}\right)}\right] \\
& =\left[\frac{\left(\mathbf{T}_{\mu}^{e} f_{n}\right) \times\left(\kappa_{n}\right)}{\left(\varphi_{n}\right) \times\left(\phi_{n}\right)}\right]=\left[\frac{\left(\mathbf{T}_{\mu}^{e} f_{n}\right)}{\left(\varphi_{n}\right)}\right] \times\left[\frac{\left(\kappa_{n}\right)}{\left(\phi_{n}\right)}\right] .
\end{aligned}
$$

Thus we get that $\overrightarrow{\mathbf{T}_{\mu}^{e}}\left(\beta_{1} * \beta_{2}\right)=\overrightarrow{\mathbf{T}_{\mu}^{e}}\left(\beta_{1}\right) \times \beta_{2}$.

This completes the proof.

Theorem 12. $\overrightarrow{\mathbf{T}}_{\mu}^{\mathrm{e}}$ defines a linear mapping from $\boldsymbol{\delta}\left(\mathbf{l}^{1},(\boldsymbol{\kappa}, *)\right.$, $*, \Delta)$ into $\boldsymbol{\delta}\left(\mathbf{1}^{1},(\boldsymbol{\kappa}, *), \times, \Delta\right)$.

The proof is straightforward.

Definition 13. Let $\beta \in \boldsymbol{\delta}\left(\mathbf{1}^{1},(\boldsymbol{\kappa}, *), \times, \Delta\right)$ be such that $\beta=$ $\left[\left(\mathbf{T}_{\mu}^{e} f_{n}\right) /\left(\phi_{n}\right)\right]$. Then we define the inverse transform of $\mathcal{T}_{\mu}^{e}$ as

$$
\left(\overrightarrow{\mathbf{T}_{\mu}^{e}}\right)^{-1}\left[\frac{\left(\mathbf{T}_{\mu}^{e} f_{n}\right)}{\left(\phi_{n}\right)}\right]=\left[\frac{\left(f_{n}\right)}{\left(\phi_{n}\right)}\right]
$$

for each $\left(\phi_{n}\right) \in \Delta$.

Theorem 14. $\overrightarrow{\mathbf{T}}_{\mu}^{\mathrm{e}}$ defines an isomorphism from $\boldsymbol{\delta}\left(\mathbf{1}^{1},(\boldsymbol{\kappa}, *)\right.$, $*, \Delta)$ onto $\delta\left(\mathbf{l}^{1},(\boldsymbol{\kappa}, *), \times, \Delta\right)$.

Proof. Assume that $\left[\left(\mathbf{T}_{\mu}^{e} f_{n}\right) /\left(\phi_{n}\right)\right]=\left[\left(\mathbf{T}_{\mu}^{e} g_{n}\right) /\left(\psi_{n}\right)\right]$ in $\boldsymbol{\delta}\left(\mathbf{1}^{1},(\boldsymbol{\kappa}\right.$, $*), \times, \Delta)$. Using (29) and Theorem 9 we get $\mathbf{T}_{\mu}^{e} f_{n} \times \psi_{m}=$ $\mathbf{T}_{\mu}^{e} g_{m} \times \phi_{n}$. Once again, Theorem 9 implies $\mathbf{T}_{\mu}^{e}\left(f_{n} * \psi_{m}\right)=$ $\mathbf{T}_{\mu}^{e}\left(g_{m} * \phi_{n}\right)$. Hence $f_{n} * \psi_{m}=g_{m} * \phi_{n}$. Therefore, $\left[\left(f_{n}\right) /\left(\phi_{n}\right)\right]=$ $\left[\left(g_{n}\right) /\left(\psi_{n}\right)\right]$. 
Now, let $\left[\left(\mathbf{T}_{\mu}^{e} f_{n}\right) /\left(\phi_{n}\right)\right] \in \boldsymbol{\delta}\left(\mathbf{1}^{1},(\boldsymbol{\kappa}, *), \times, \Delta\right)$; then $\mathbf{T}_{\mu}^{e} f_{n} \times$ $\phi_{m}=\mathbf{T}_{\mu}^{e} f_{m} \times \phi_{n}, \forall m, n \in \mathbb{N}$. Theorem 9 leads to $\mathbf{T}_{\mu}^{e}\left(f_{n} *\right.$ $\left.\phi_{m}\right)=\mathbf{T}_{\mu}^{e}\left(f_{m} * \phi_{n}\right)$. Hence $\left[\left(f_{n}\right) /\left(\phi_{n}\right)\right] \in \boldsymbol{\delta}\left(\mathbf{l}^{1},(\boldsymbol{\kappa}, *), *, \Delta\right)$ is the Boehmian that satisfies $\overrightarrow{\mathbf{T}_{\mu}^{e}}\left[\left(f_{n}\right) /\left(\phi_{n}\right)\right]=\left[\left(\mathbf{T}_{\mu}^{e} f_{n}\right) /\left(\phi_{n}\right)\right]$.

This completes the proof of the theorem.

Theorem 15. Let $\left[\left(f_{n}\right) /\left(\phi_{n}\right)\right] \in \boldsymbol{\delta}\left(\mathbf{1}^{1},(\boldsymbol{\kappa}, *), *, \Delta\right)$ and $\phi \in$ $\boldsymbol{\kappa}\left(\mathbb{R}^{2}\right)$; then

$$
\overrightarrow{\mathbf{T}_{\mu}^{e}}\left(\left[\frac{\left(f_{n}\right)}{\left(\phi_{n}\right)}\right] * \phi\right)=\left[\frac{\left(\mathbf{T}_{\mu}^{e} f_{n}\right)}{\left(\phi_{n}\right)}\right] \times \phi .
$$

Proof. Applying Definition 10 for each $\left[\left(f_{n}\right) /\left(\phi_{n}\right)\right] \in \boldsymbol{\delta}\left(\mathbf{l}^{1}\right.$, $(\boldsymbol{\kappa}, *), \times, \Delta)$ and $\phi \in \boldsymbol{\kappa}\left(\mathbb{R}^{2}\right)$ yields

$$
\left(\overrightarrow{\mathbf{T}_{\mu}^{e}}\right)\left(\left[\frac{\left(f_{n}\right)}{\left(\phi_{n}\right)}\right] * \phi\right)=\left[\frac{\left(\mathbf{T}_{\mu}^{e}\right)\left(\left(f_{n}\right) * \phi\right)}{\left(\phi_{n}\right)}\right] .
$$

By Theorem 9 we get

$$
\left(\overrightarrow{\mathbf{T}_{\mu}^{e}}\right)\left(\left[\frac{\left(f_{n}\right)}{\left(\phi_{n}\right)}\right] * \phi\right)=\left[\frac{\left(f_{n}\right) \times \phi}{\left(\phi_{n}\right)}\right]=\left[\frac{\left(f_{n}\right)}{\left(\phi_{n}\right)}\right] \times \phi .
$$

This completes the proof of the theorem.

Theorem 16. The mappings $\overrightarrow{\mathbf{T}_{\mu}^{e}}$ and $\left(\overrightarrow{\mathbf{T}}_{\mu}^{e}\right)$ are continuous with respect to $\delta$ and $\Delta$ convergence.

Proof. First of all, we show that $\overrightarrow{\mathbf{T}}_{\mu}^{\mathrm{e}}$ and $\left(\overrightarrow{\mathbf{T}}_{\mu}^{\mathrm{e}}\right)$ are continuous with respect to $\delta$ convergence.

Let $\beta_{n} \stackrel{\delta}{\rightarrow} \beta$ in $\boldsymbol{\delta}\left(\mathbf{l}^{1},(\boldsymbol{\kappa}, *), *, \Delta\right)$ as $n \rightarrow \infty$; then we show that $\overrightarrow{\mathbf{T}_{\mu}^{e}} \beta_{n} \rightarrow \overrightarrow{\mathbf{T}_{\mu}^{e}} \beta$ as $n \rightarrow \infty$. By virtue of Theorem 1 we can find $f_{n, k}$ and $f_{k}$ in $\mathbf{l}^{1}\left(\mathbb{R}^{2}\right)$ such that $\beta_{n}=\left[f_{n, k} / \phi_{k}\right]$ and $\beta=\left[f_{k} / \phi_{k}\right]$ such that $f_{n, k} \rightarrow f_{k}$ as $n \rightarrow \infty$ for every $k \in \mathbb{N}$.

Employing the continuity condition of $\mathbf{T}_{\mu}^{e}$ transform implies $\mathbf{T}_{\mu}^{e} f_{n, k} \rightarrow \mathbf{T}_{\mu}^{e} f_{k}$ as $n \rightarrow \infty$ in the space $\mathbf{l}^{1}\left(\mathcal{S}^{1} \times \mathbb{R}\right)$.

Thus,

$$
\left[\frac{\mathbf{T}_{\mu}^{e} f_{n, k}}{\phi_{k}}\right] \longrightarrow\left[\frac{\mathbf{T}_{\mu}^{e} f_{k}}{\phi_{k}}\right] \text { as } n \longrightarrow \infty
$$

in $\boldsymbol{\delta}\left(\mathbf{1}^{1},(\boldsymbol{\kappa}, *), \times, \Delta\right)$.

To prove the second part, let $g_{n} \stackrel{\delta}{\rightarrow} g$ in $\boldsymbol{\delta}\left(\mathbf{l}^{1},(\boldsymbol{\kappa}, *), \times, \Delta\right)$ as $n \rightarrow \infty$. Then, once again, by Theorem $1, g_{n}=\left[\mathbf{T}_{\mu}^{e} f_{n, k} / \phi_{k}\right]$ and $g=\left[\mathbf{T}_{\mu}^{e} f_{k} / \phi_{k}\right]$ and $\mathbf{T}_{\mu}^{e} f_{n, k} \rightarrow \mathbf{T}_{\mu}^{e} f_{k}$ as $n \rightarrow \infty$. Hence $f_{n, k} \rightarrow f_{k}$ in $\boldsymbol{\delta}\left(\mathbf{l}^{1},(\boldsymbol{\kappa}, *), *, \Delta\right)$ as $n \rightarrow \infty$. That is, $\left[f_{n, k} / \phi_{k}\right] \rightarrow\left[f_{k} / \phi_{k}\right]$ as $n \rightarrow \infty$. Using (33) we get

$$
\left(\overrightarrow{\mathbf{T}_{\mu}^{e}}\right)^{-1}\left[\frac{\mathbf{T}_{\mu}^{e} f_{n, k}}{\phi_{k}}\right] \longrightarrow\left(\overrightarrow{\mathbf{T}_{\mu}^{e}}\right)^{-1}\left[\frac{\mathbf{T}_{\mu}^{e} f_{k}}{\phi_{k}}\right] \quad \text { as } n \longrightarrow \infty .
$$

Now, we establish continuity of $\overrightarrow{\mathbf{T}_{\mu}^{e}}$ and $\left(\overrightarrow{\mathbf{T}}_{\mu}^{e}\right)^{-1}$ with respect to $\Delta$ convergence.
Let $\beta_{n}, \beta \in \boldsymbol{\delta}\left(\mathbf{l}^{1},(\boldsymbol{\kappa}, *), *, \Delta\right)$ be such that $\beta_{n} \stackrel{\Delta}{\rightarrow} \beta$ as $n \rightarrow$ $\infty$. Then, by Theorem 1 we can find that $f_{n} \in \mathbf{l}^{1}\left(\mathcal{S}^{1} \times \mathbb{R}\right)$ and $\left(\phi_{n}\right) \in \Delta$ such that $\left(\beta_{n}-\beta\right) * \phi_{n}=\left[\left(\left(f_{n}\right) * \phi_{k}\right) / \phi_{k}\right]$ and $f_{n} \rightarrow 0$ as $n \rightarrow \infty$. Employing (29) we get

$$
\overrightarrow{\mathbf{T}_{\mu}^{e}}\left(\left(\beta_{n}-\beta\right) * \phi_{n}\right)=\left[\frac{\mathbf{T}_{\mu}^{e}\left(\left(f_{n}\right) * \phi_{k}\right)}{\phi_{k}}\right] .
$$

Hence, we have $\overrightarrow{\mathbf{T}_{\mu}^{e}}\left(\left(\beta_{n}-\beta\right) * \phi_{n}\right)=\left[\left(\left(\mathbf{T}_{\mu}^{e} f_{n}\right) \times \phi_{k}\right) / \phi_{k}\right]=$ $\mathbf{T}_{\mu}^{e} f_{n} \rightarrow 0$ as $n \rightarrow \infty$ in $\mathbf{l}^{1}\left(\mathcal{S}^{1} \times \mathbb{R}\right)$.

Therefore

$$
\begin{aligned}
\overrightarrow{\mathbf{T}_{\mu}^{e}}\left(\left(\beta_{n}-\beta\right) * \phi_{n}\right) & =\left(\overrightarrow{\mathbf{T}_{\mu}^{e}} \beta_{n}-\overrightarrow{\mathbf{T}_{\mu}^{e}} \beta\right) \times \phi_{n} \\
& \Longrightarrow \text { as } n \longrightarrow \infty .
\end{aligned}
$$

Hence, $\overrightarrow{\mathbf{T}_{\mu}^{\mathrm{e}}} \beta_{n} \stackrel{\Delta}{\rightarrow} \overrightarrow{\mathbf{T}_{\mu}^{\mathrm{e}}} \beta$ as $n \rightarrow \infty$.

Finally, let $g_{n} \stackrel{\Delta}{\rightarrow} g$ in $\boldsymbol{\delta}\left(\mathbf{1}^{1},(\boldsymbol{\kappa}, *), *, \Delta\right)$ as $n \rightarrow \infty$; then we find $\mathbf{T}_{\mu}^{e} f_{k} \in \mathbf{l}^{1}\left(\mathcal{S}^{1} \times \mathbb{R}\right)$ such that $\left(g_{n}-g\right) \times \phi_{n}=\left[\left(\mathbf{T}_{\mu}^{e} f_{k} \times\right.\right.$ $\left.\left.\phi_{k}\right) / \phi_{k}\right]$ and $\mathbf{T}_{\mu}^{e} f_{k} \rightarrow 0$ as $n \rightarrow \infty$ for some $\left(\phi_{n}\right) \in \Delta$.

Now, using (33), we obtain

$$
\left(\overrightarrow{\mathbf{T}_{\mu}^{e}}\right)^{-1}\left(\left(g_{n}-g\right) \times \phi_{n}\right)=\left[\frac{\left(\mathbf{T}_{\mu}^{e}\right)^{-1}\left(\mathbf{T}_{\mu}^{e} f_{k} \times \phi_{k}\right)}{\phi_{k}}\right] \text {. }
$$

Theorem 9 implies

$$
\begin{aligned}
& \left(\overrightarrow{\mathbf{T}_{\mu}^{e}}\right)^{-1}\left(\left(g_{n}-g\right) \times \phi_{n}\right)=\left[\frac{\left(f_{n}\right) * \phi_{k}}{\phi_{k}}\right] \\
& \quad=f_{n} \longrightarrow 0 \in \mathbf{l}^{1}\left(\mathcal{S}^{1} \times \mathbb{R}\right) \text { as } n \longrightarrow \infty .
\end{aligned}
$$

Thus

$$
\begin{array}{r}
\left(\overrightarrow{\mathbf{T}_{\mu}^{\mathrm{e}}}\right)^{-1}\left(\left(g_{n}-g\right) \times \phi_{n}\right) \\
=\left(\left(\overrightarrow{\mathbf{T}_{\mu}^{\mathrm{e}}}\right)^{-1} g_{n}-\left(\overrightarrow{\mathbf{T}_{\mu}^{e}}\right)^{-1} g\right) * \phi_{n} \rightarrow 0 \in \mathbf{l}^{1}\left(\mathcal{\delta}^{1} \times \mathbb{R}\right) \\
\text { as } n \longrightarrow \infty .
\end{array}
$$

From this we find that $\left(\overrightarrow{\mathbf{T}}_{\mu}^{e}\right)^{-1} g_{n} \stackrel{\Delta}{\rightarrow}\left(\overrightarrow{\mathbf{T}}_{\mu}^{e}\right)^{-1} g \in \boldsymbol{\delta}\left(\mathbf{1}^{1},(\boldsymbol{\kappa}, *)\right.$, $*, \Delta)$ as $n \rightarrow \infty$.

This completes the proof of the theorem.

Theorem 17. The transform $\overrightarrow{\mathrm{T}_{\mu}^{\mathrm{e}}}$ is consistent with $\overrightarrow{\mathrm{T}_{\mu}^{\mathrm{e}}}: \mathbf{l}^{1}\left(\mathcal{S}^{1} \times\right.$ $\mathbb{R}) \rightarrow \mathbf{l}^{1}\left(\delta^{1} \times \mathbb{R}\right)$.

Proof. For every $f \in \mathbf{l}^{1}\left(\mathcal{S}^{1} \times \mathbb{R}\right)$, let $\beta \in \boldsymbol{\delta}\left(\mathbf{l}^{1},(\boldsymbol{\kappa}, *), *, \Delta\right)$ be its representative; then $\forall n \in \mathbb{N},\left(\varphi_{n}\right) \in \Delta, \beta=\left[\left(f *\left(\varphi_{n}\right)\right) /\left(\varphi_{n}\right)\right]$. For all $n \in \mathbb{N}$ it is clear that $\left(\varphi_{n}\right)$ is independent of the representative. 
We have

$$
\begin{aligned}
\overrightarrow{\mathbf{T}_{\mu}^{e}}(\beta) & =\overrightarrow{\mathbf{T}_{\mu}^{e}}\left(\left[\frac{f *\left(\varphi_{n}\right)}{\left(\varphi_{n}\right)}\right]\right)=\left[\frac{\mathbf{T}_{\mu}^{e}\left(f *\left(\varphi_{n}\right)\right)}{\left(\varphi_{n}\right)}\right] \\
& =\left[\frac{\mathbf{T}_{\mu}^{e} f \times\left(\varphi_{n}\right)}{\left(\varphi_{n}\right)}\right]
\end{aligned}
$$

which is the representative of $\mathbf{T}_{\mu}^{e} f$ in the space $\mathbf{l}^{1}\left(\mathcal{S}^{1} \times \mathbb{R}\right)$.

Hence the proof is completed.

Theorem 18. The necessary and sufficient condition for $\left[\left(g_{n}\right) /\left(\psi_{n}\right)\right] \in \boldsymbol{\delta}\left(\mathbf{l}^{1},(\boldsymbol{\kappa}, *), \times, \Delta\right)$ to be in the range of $\overrightarrow{\mathbf{T}_{\mu}^{e}}$ is that $g_{n}$ belongs to range of $\mathbf{T}_{\mu}^{e}$ for every $n \in \mathbb{N}$.

Proof. Let $\left[\left(g_{n}\right) /\left(\psi_{n}\right)\right]$ be in the range of $\overrightarrow{\mathbf{T}}_{\mu}^{\mathrm{e}}$; then of course $g_{n}$ belongs to the range of $\mathbf{T}_{\mu}^{e}, \forall n \in \mathbb{N}$.

To establish the converse, let $g_{n}$ be in the range of $\mathbf{T}_{\mu}^{e}$, $\forall n \in \mathbb{N}$. Then there is $f_{n} \in \mathbf{1}^{1}\left(\mathcal{S}^{1} \times \mathbb{R}\right)$ such that $\mathbf{T}_{\mu}^{e} f_{n}=$ $g_{n}, n \in \mathbb{N}$.

Since $\left[\left(g_{n}\right) /\left(\psi_{n}\right)\right] \in \boldsymbol{\delta}\left(\mathbf{1}^{1},(\boldsymbol{\kappa}, *), \times, \Delta\right)$ we get $g_{n} \times \psi_{m}=$ $g_{m} \times \psi_{n}, \forall m, n \in \mathbb{N}$.

Therefore, Theorem 9 yields

$$
\mathbf{T}_{\mu}^{e}\left(f_{n} * \varphi_{n}\right)=\mathbf{T}_{\mu}^{e}\left(f_{m} * \varphi_{n}\right), \quad \forall m, n \in \mathbb{N},
$$

where $f_{n} \in \mathbf{l}^{1}\left(\mathcal{S}^{1} \times \mathbb{R}\right)$ and $\varphi_{n} \in \Delta, \forall n \in \mathbb{N}$.

Thus $f_{n} * \varphi_{m}=f_{m} * \varphi_{n}, m, n \in \mathbb{N}$. Hence,

$$
\begin{aligned}
& {\left[\frac{\left(f_{n}\right)}{\left(\varphi_{n}\right)}\right] \in \boldsymbol{\delta}\left(\mathbf{1}^{1},(\boldsymbol{\kappa}, *), *, \Delta\right),} \\
& \overrightarrow{\mathbf{T}_{\mu}^{e}}\left(\left[\frac{\left(f_{n}\right)}{\left(\varphi_{n}\right)}\right]\right)=\left[\frac{\left(g_{n}\right)}{\left(\psi_{n}\right)}\right] .
\end{aligned}
$$

The theorem is therefore completely proved.

\section{Conflict of Interests}

The authors declare that they have no conflict of interests regarding publication of this paper.

\section{Acknowledgment}

The second author gratefully acknowledges the partial support that this research received from the Universiti Putra Malaysia under GP-IBT Grant Scheme having Project no. GPIBT/2013/9420100.

\section{References}

[1] E. A. Robinson, "Spectral approach to geophysical inversion by Lorentz, Fourier and Radon transforms," Proceedings of the IEEE Institute of Electrical and Electronics Engineers, vol. 70, no. 9, pp. 1039-1054, 1982.

[2] C. H. Chapman, "Generalized Radon transforms and slant stacks," Geophysical Journal, Royal Astronomical Society, vol. 66, no. 2, pp. 445-453, 1981.
[3] P. Mikusiński and A. Zayed, "The Radon transform of Boehmians," Proceedings of the American Mathematical Society, vol. 118, no. 2, pp. 561-570, 1993.

[4] R. Roopkumar, "Generalized Radon transform," The Rocky Mountain Journal of Mathematics, vol. 36, no. 4, pp. 1375-1390, 2006.

[5] D. Ludwig, "The Radon transform on euclidean space," Communications on Pure and Applied Mathematics, vol. 19, pp. 4981, 1966.

[6] S. R. Deans, The Radon Transform and Some of Its Applications, John Wiley \& Sons, New York, NY, USA, 1973.

[7] Á. Kurusa, "A characterization of the Radon transform and its dual of Euclidean space," Acta Universitatis Szegediensis, vol. 54, no. 3-4, pp. 273-276, 1990.

[8] A. Hertle, "A characterization of Fourier and Radon transforms on Euclidean space," Transactions of the American Mathematical Society, vol. 273, no. 2, pp. 595-607, 1982.

[9] A. Hertle, "Continuity of the Radon transform and its inverse on Euclidean space," Mathematische Zeitschrift, vol. 184, no. 2, pp. 165-192, 1983.

[10] G. Beylkin, "Discrete Radon transform," Institute of Electrical and Electronics Engineers. Transactions on Acoustics, Speech, and Signal Processing, vol. 35, no. 2, pp. 162-172, 1987.

[11] P. Fishburn, P. Schwander, L. Shepp, and R. J. Vanderbei, “The discrete Radon transform and its approximate inversion via linear programming," Discrete Applied Mathematics, vol. 75, no. 1, pp. 39-61, 1997.

[12] A. V. Clough and H. H. Barrett, "Attenuated radon and abel transforms," Journal of the Optical Society of America, vol. 73, no. 11, pp. 1590-1595, 1983.

[13] G. Bal and P. Moireau, "Fast numerical inversion of the attenuated Radon transform with full and partial measurements," Inverse Problems, vol. 20, no. 4, pp. 1137-1164, 2004.

[14] C. E. Yarman and B. Yazici, "A new exact inversion method for exponential Radon transform using the harmonic analysis of the Euclidean motion group," Inverse Problems and Imaging, vol. 1, no. 3, pp. 457-479, 2007.

[15] E. Y. Sidky, C.-M. Kao, P. J. LaRiviere, and X. Pan, "Noise properties of the inverse $\pi$-scheme exponential radon transform," in Medical Imaging: Image Processing, vol. 4684 of Proceedings of SPIE, pp. 790-800, February 2002.

[16] O. Tretiak and C. Metz, "The exponential Radon transform," SIAM Journal on Applied Mathematics, vol. 39, no. 2, pp. 341354, 1980.

[17] S. K. Q. Al-Omari, "Hartley transforms on a certain space of generalized functions," Georgian Mathematical Journal, vol. 20, no. 3, pp. 415-426, 2013.

[18] S. K. Q. Al-Omari and A. Kılıçman, "Note on Boehmians for class of optical Fresnel wavelet transforms," Journal of Function Spaces and Applications, vol. 2012, Article ID 405368, 14 pages, 2012.

[19] S. K. Q. Al-Omari and A. Kılıçman, "On generalized HartleyHilbert and Fourier-Hilbert transforms," Advances in Difference Equations, vol. 2012, article 232, 12 pages, 2012.

[20] T. K. Boehme, “The support of Mikusiński operators," Transactions of the American Mathematical Society, vol. 176, pp. 319-334, 1973.

[21] S. K. Q. Al-Omari and A. K1lıçman, "On diffraction Fresnel transforms for Boehmians," Abstract and Applied Analysis, vol. 2011, Article ID 712746, 11 pages, 2011. 


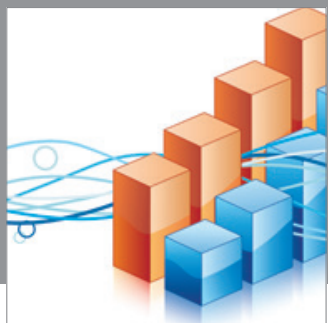

Advances in

Operations Research

mansans

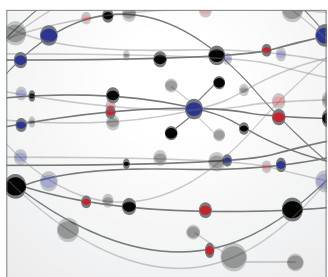

The Scientific World Journal
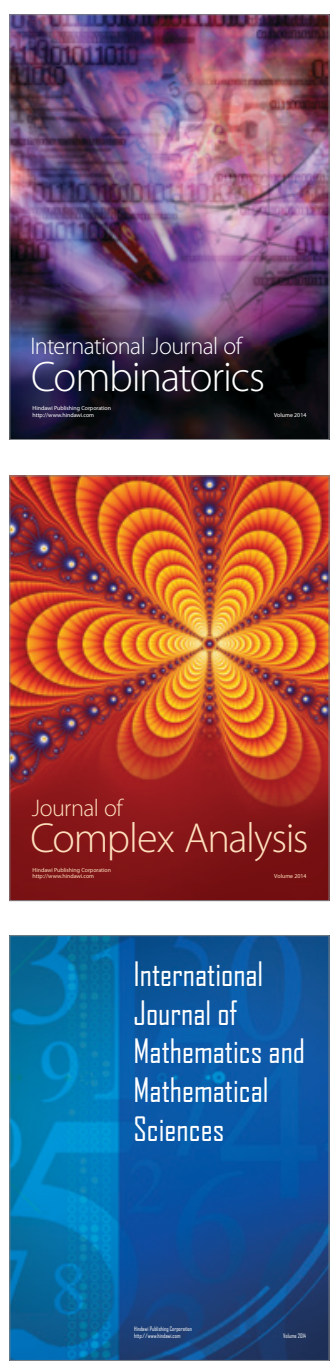
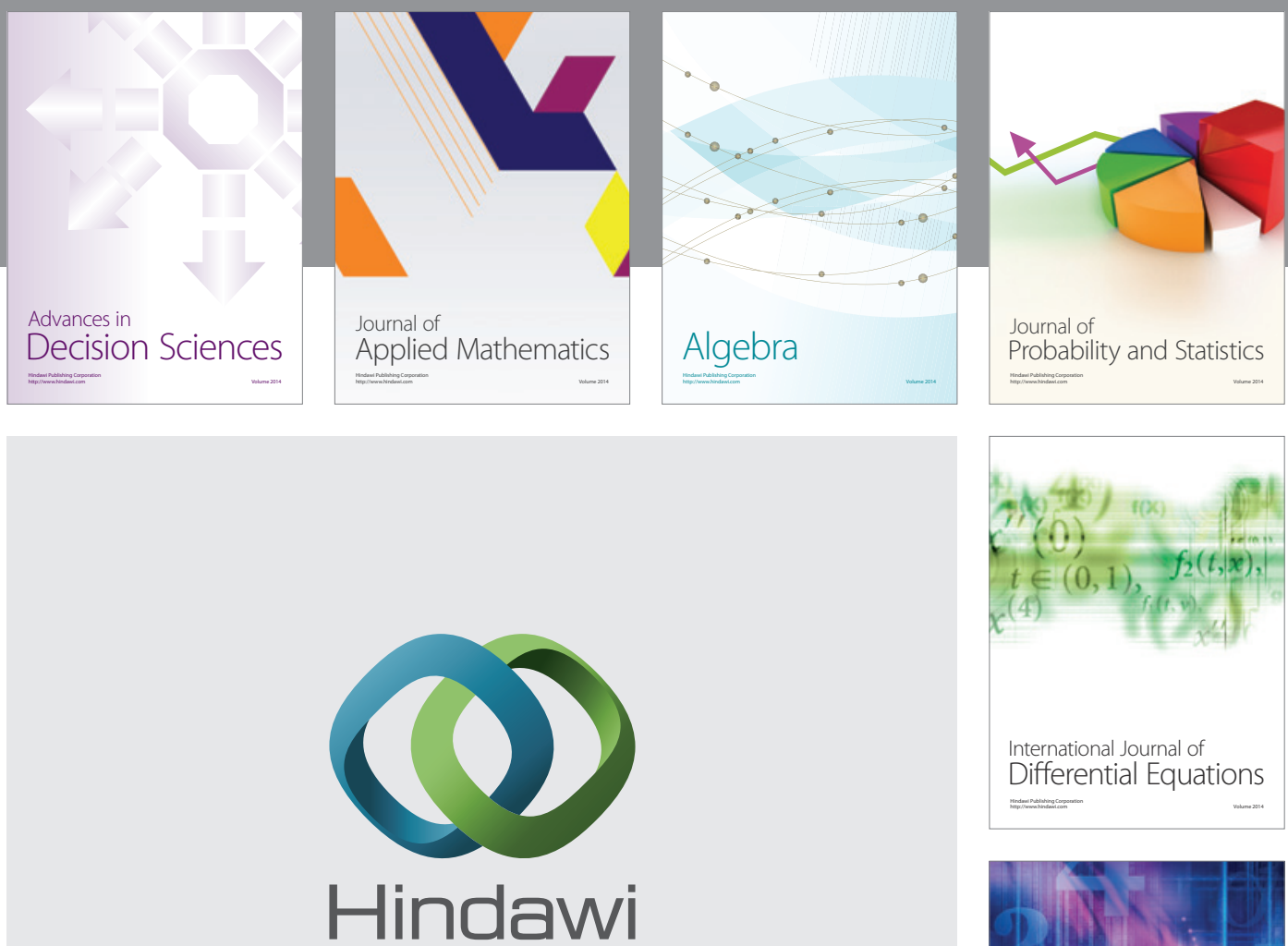

Submit your manuscripts at http://www.hindawi.com
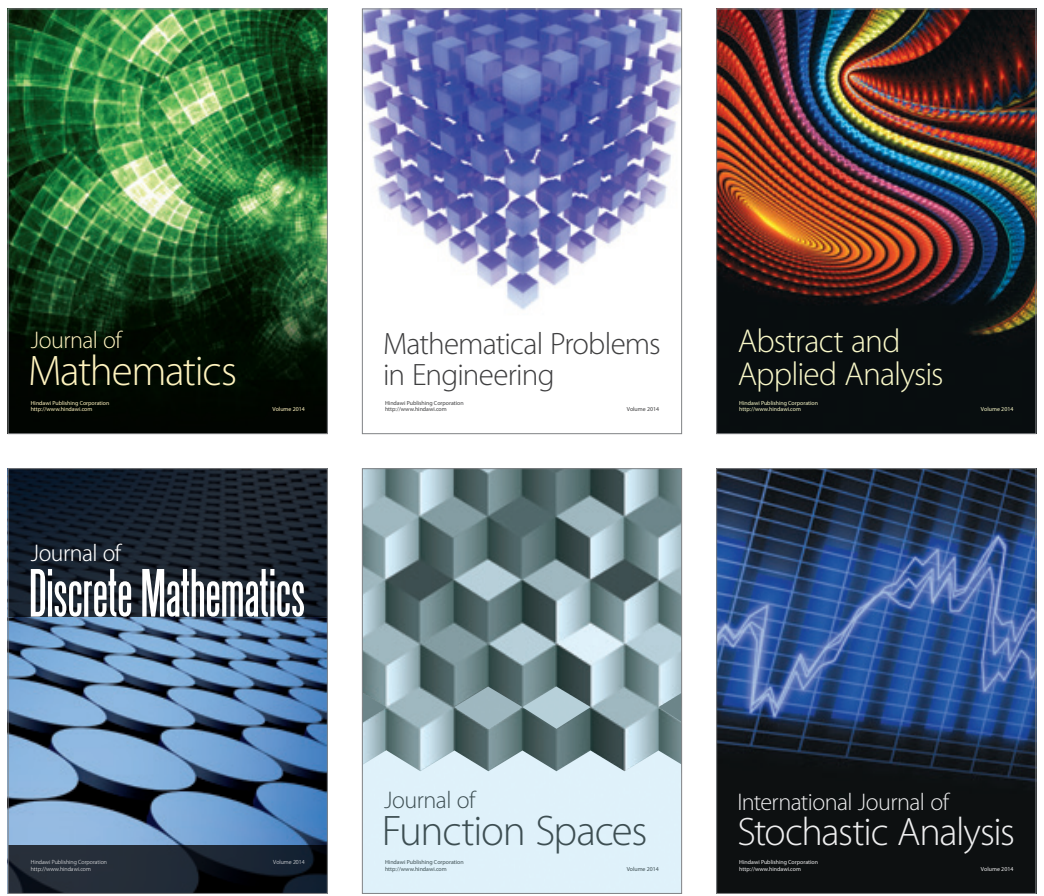

Journal of

Function Spaces

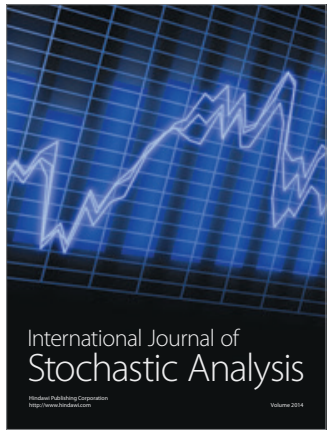

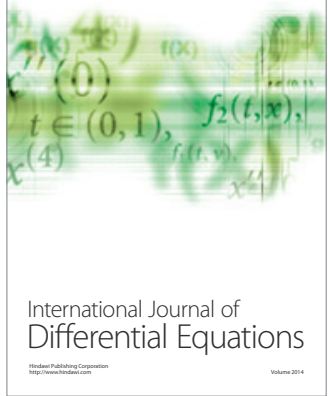
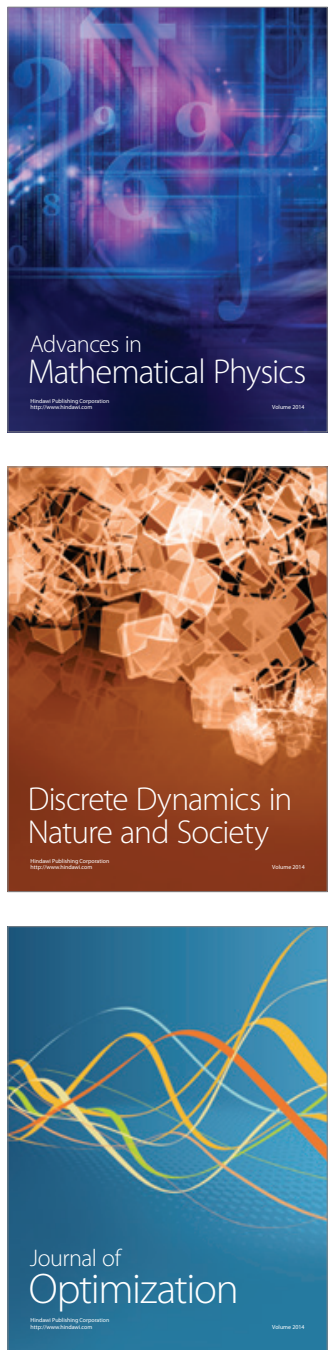